\title{
Termination-dependent diversity performance of coupled antennas: Network theory analysis
}

Michael A. Jensen

jensen@byu.edu

Jon W. Wallace

wall@ieee.org

Follow this and additional works at: https://scholarsarchive.byu.edu/facpub

Part of the Electrical and Computer Engineering Commons

\section{Original Publication Citation}

Wallace, J. W., and M. A. Jensen. "Termination-Dependent Diversity Performance of Coupled Antennas: Network Theory Analysis." Antennas and Propagation, IEEE Transactions on 52.1 (24): 98-15

\section{BYU ScholarsArchive Citation}

Jensen, Michael A. and Wallace, Jon W., "Termination-dependent diversity performance of coupled antennas: Network theory analysis" (2004). Faculty Publications. 460.

https://scholarsarchive.byu.edu/facpub/460 accepted for inclusion in Faculty Publications by an authorized administrator of BYU ScholarsArchive. For more information, please contact ellen_amatangelo@byu.edu. 


\title{
Termination-Dependent Diversity Performance of Coupled Antennas: Network Theory Analysis
}

\author{
Jon W. Wallace, Member, IEEE, and Michael A. Jensen, Senior Member, IEEE
}

\begin{abstract}
A new analysis of mutually coupled diversity antenna systems is presented that includes the effects of coupling on the radiation pattern and received power. This analysis, based upon the network scattering parameters, makes use of the singular value decomposition to both specify the characteristics of and analyze terminated antenna networks. The approach facilitates a simplified proof of the multiport conjugate matching condition as well as development of expressions for diversity performance as a function of various impedance terminations. Application of the analysis to coupled dipole antennas characterized using full-wave electromagnetic analysis leads to a comprehensive examination of the diversity performance obtainable for such antennas under different termination conditions. The results from this paper reveal that for closely spaced antennas, the termination can play a noticeable role in determining the diversity gain offered by coupled antennas.
\end{abstract}

Index Terms-Antenna array mutual coupling, diversity methods, impedance matching.

\section{INTRODUCTION}

A NTENNA arrays play a crucial role in wireless communications over multipath fading channels, with antenna diversity being the topic of considerable research for many decades [1]. When using multiple antenna elements for diversity implementation on small personal communications devices, the resulting closely spaced antenna elements exhibit the well-known mutual coupling [2], which alters both their terminal impedance and radiation pattern characteristics. These changes obviously impact the diversity performance of the multiantenna system.

A variety of studies have appeared that examine the diversity performance of coupled antennas. Most such studies have emphasized the effect of coupling on the antenna radiation pattern and the resulting correlation between the received signals [3]-[8]. Generally, these studies neglect the impact of the altered impedance on the received power. Even when this effect is included in the analysis, typically only a single termination [9]-[11] or a limited set of terminations [12] is considered. As of yet, there does not appear to be a comprehensive study including both the pattern and power implications of mutual coupling for different termination conditions, particularly optimal antenna matching schemes.

Manuscript received December 9, 2002; revised March 18, 2003. This work was supported by the National Science Foundation under Wireless Initiative Grant CCR 99-79452 and Information Technology Research Grant CCR-0081476.

The authors are with the Department of Electrical and Computer Engineering, Brigham Young University, Provo, UT 84602 USA (e-mail: jensen@ee.byu.edu).

Digital Object Identifier 10.1109/TAP.2003.822444

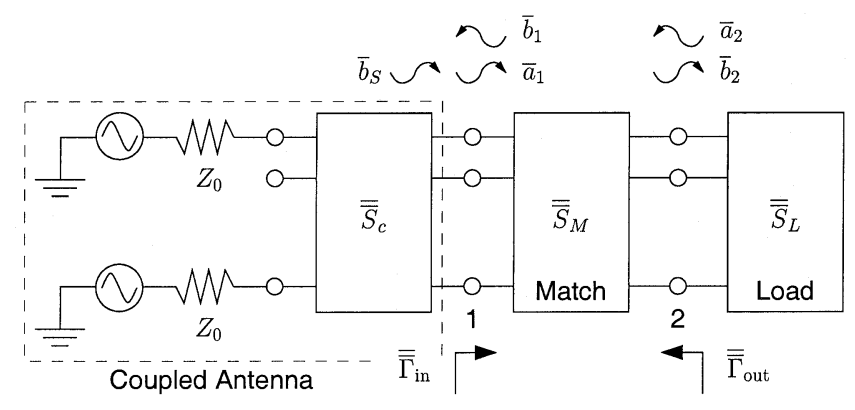

Fig. 1. System model of a coupled diversity antenna array connected to a multiport matching circuit and individual loads.

In this paper, we present a framework that includes the impact of antenna termination in assessing the diversity performance of coupled systems. The analysis exploits singular value decompositions (SVDs) of the scattering-parameter (S-parameter) [13] representations of the antenna, matching network, and load. The framework facilitates a simple proof of the multiport conjugate matching condition [10], [14]-[16] and reveals that proper termination not only maximizes received power but also can perfectly decorrelate the received signals. Furthermore, the methodology provides new tools for multiantenna or multiport system analysis and allows mathematical definition of the multiport optimal matching network. Computational results for mutually coupled dipoles demonstrate the achievable diversity performance for different antenna terminations. These results indicate that, for close spacing, the termination can play a key role in the achievable diversity performance of the system.

\section{Coupled Antenna Network Representation}

\section{A. S-Parameter Network Description}

For high-frequency systems such as mutually coupled antenna networks, the S-parameter matrix representation [13] provides a convenient analysis framework. In this description, the voltages and currents on each of the $N$ ports are decomposed into inward $(\bar{a})$ and outward $(\bar{b})$ traveling waves that satisfy the relation $\bar{b}=\overline{\bar{S}} \bar{a}$, where $\overline{\bar{S}}$ is the $N \times N$ S-parameter matrix or $S$-matrix. The voltage on $\left(v_{n}\right)$ and current into $\left(i_{n}\right)$ the $n$th port are related to $a_{n}$ and $b_{n}$ according to

$$
\begin{aligned}
& v_{n}=Z_{0}^{1 / 2}\left(a_{n}+b_{n}\right) \\
& i_{n}=Z_{0}^{-1 / 2}\left(a_{n}-b_{n}\right)
\end{aligned}
$$

where $Z_{0}$ is a normalizing impedance.

Consider now the network depiction of the coupled receiving antenna system shown in Fig. 1. In this diagram, each element of 
the coupled array is characterized by a generator whose signal passes through a coupling matrix $\overline{\bar{S}}_{c}$ with a block representation

$$
\overline{\bar{S}}_{c}=\left[\begin{array}{ll}
\overline{\bar{S}}_{c, 11} & \overline{\bar{S}}_{c, 12} \\
\overline{\bar{S}}_{c, 21} & \overline{\bar{S}}_{c, 22}=\overline{\bar{S}}_{S}
\end{array}\right]
$$

where "1" and "2" refer to input and output ports, respectively. Here, we have used the notation $\overline{\bar{S}}_{c, 22}=\overline{\bar{S}}_{S}$ to emphasize that this block represents the traditional "source termination" encountered in S-parameter circuit analysis. It is also noteworthy that $\overline{\bar{S}}_{S}$ is the coupled S-parameter matrix measured at the antenna element input ports. Rather than trying to characterize the remaining blocks of $\bar{S}_{c}$, we will simply represent the excitation signal at the antenna ports as $\bar{b}_{S}$ so that

$$
\bar{a}_{1}=\overline{\bar{S}}_{S} \bar{b}_{1}+\bar{b}_{S}
$$

The $N$-port antenna in Fig. 1 is attached to the $M$-port load network $\bar{S}_{L}$ through a matching network with S-parameter matrix $\overline{\bar{S}}_{M}$ consisting of blocks $\overline{\bar{S}}_{i j}, i, j \in 1,2$, arranged in a form similar to that in (2). Here, we will consider networks having the same number of input and output ports $(M=N)$. We briefly discuss implications of $M \neq N$ in Section III.

For the network in Fig. 1, the input and output reflection coefficients can be expressed as

$$
\begin{aligned}
\overline{\bar{\Gamma}}_{\text {in }} & =\overline{\bar{S}}_{11}+\overline{\bar{S}}_{12}\left(\overline{\bar{I}}-\overline{\bar{S}}_{L} \overline{\bar{S}}_{22}\right)^{-1} \overline{\bar{S}}_{L} \overline{\bar{S}}_{21} \\
& =\overline{\bar{S}}_{11}+\overline{\bar{S}}_{12} \overline{\bar{S}}_{L}\left(\overline{\bar{I}}_{-}-\overline{\bar{S}}_{22} \overline{\bar{S}}_{L}\right)^{-1} \overline{\bar{S}}_{21} \\
\overline{\bar{\Gamma}}_{\text {out }} & =\overline{\bar{S}}_{22}+\overline{\bar{S}}_{21}\left(\overline{\bar{I}}-\overline{\bar{S}}_{S} \overline{\bar{S}}_{11}\right)^{-1} \overline{\bar{S}}_{S} \overline{\bar{S}}_{12}
\end{aligned}
$$

where the two forms for $\overline{\bar{\Gamma}}_{\text {in }}$ are equivalent.

\section{B. SVD Network Representation}

The SVD of the S-parameter matrices facilitates analysis and provides a useful interpretation of the circuit's fundamental behavior. This decomposition is given by $\overline{\bar{S}}=\overline{\overline{U \Lambda}}^{1 / 2} \overline{\bar{V}}^{H}$, where $\overline{\bar{U}}$ and $\overline{\bar{V}}$ are unitary matrices of singular vectors, $\bar{\Lambda}^{1 / 2}$ is a diagonal matrix of corresponding ordered singular values (largest to smallest), and $\{\cdot\}^{H}$ is the conjugate transpose operation. We may therefore write the $S$-matrix relation $\bar{b}=\overline{\bar{S}} \bar{a}$ as

$$
\underbrace{\overline{\bar{U}}^{H} \bar{b}}_{\bar{b}^{\prime}}=\overline{\bar{\Lambda}}^{1 / 2} \underbrace{\overline{\bar{V}}^{H} \bar{a}}_{\bar{a}^{\prime}} \text {. }
$$

Considering vectors $\bar{b}^{\prime}$ and $\bar{a}^{\prime}$, the response of the network is now characterized by $N$ one-port elements, where the $n$th element presents a positive reflection coefficient $\Lambda_{n n}^{1 / 2}$.

Network loss characteristics are an important consideration for matching networks. Consider the net power delivered to a network that, using (7), is given as

$$
\begin{aligned}
P_{\text {net }}=\|\bar{a}\|^{2}-\|\bar{b}\|^{2} & =\bar{a}^{H}\left(\overline{\bar{I}}-\overline{\bar{S}}^{H} \overline{\bar{S}}\right) \bar{a} \\
& =\sum_{n}\left(1-\lambda_{n}\right)\left|a_{n}^{\prime}\right|^{2}
\end{aligned}
$$

where $\lambda_{n}=\Lambda_{n n}$. Thus, the network loss behavior is primarily defined by $\overline{\bar{\Lambda}}$. For lossless networks, $P_{\text {net }}=0$, so that $\lambda_{n}=1$ for all $n$. For lossy networks, $P_{\text {net }}>0$, so that $\lambda_{n}<1$ for all $n$.

\section{Lossless Matching Networks}

Lossless matching networks are of particular interest since they may be constructed from all passive components exhibiting low noise figures. Such networks must satisfy $\overline{\bar{S}}_{M}^{H} \overline{\bar{S}}_{M}=\overline{\bar{I}}$, resulting in the conditions

$$
\begin{aligned}
& \overline{\bar{S}}_{11}^{H} \overline{\bar{S}}_{11}+\overline{\bar{S}}_{21}^{H} \overline{\bar{S}}_{21}=\overline{\bar{I}} \\
& \overline{\bar{S}}_{11}^{H} \overline{\bar{S}}_{12}+\overline{\bar{S}}_{21}^{H} \overline{\bar{S}}_{22}=\overline{\overline{0}} \\
& \overline{\bar{S}}_{12}^{H} \overline{\bar{S}}_{12}+\overline{\bar{S}}_{22}^{H} \overline{\bar{S}}_{22}=\overline{\bar{I}} .
\end{aligned}
$$

Substitution of the SVD of the subblocks $\overline{\bar{S}}_{i j}=\overline{\bar{U}}_{i j} \overline{\bar{\Lambda}}_{i j}^{1 / 2} \overline{\bar{V}}_{i j}^{H}$ into (10) yields

$$
\begin{array}{ll}
\overline{\bar{V}}_{21} \overline{\bar{\Theta}}_{21}=\overline{\bar{V}}_{11} & \overline{\bar{\Lambda}}_{21}=\overline{\bar{I}}-\overline{\bar{\Lambda}}_{11} \\
\overline{\bar{V}}_{12} \overline{\bar{\Theta}}_{12}=\overline{\bar{V}}_{22} & \overline{\bar{\Lambda}}_{12}=\overline{\bar{I}}-\overline{\bar{\Lambda}}_{22}
\end{array}
$$

where $\overline{\bar{\Theta}}_{21}$ and $\overline{\bar{\Theta}}_{12}$ are diagonal phase shift matrices with arbitrary complex elements of unit magnitude. This operation also produces the condition

$$
\begin{aligned}
\overline{\bar{\Lambda}}_{11}^{1 / 2} \overline{\bar{U}}_{11}^{H} \overline{\bar{U}}_{12}\left(\overline{\bar{I}}-\overline{\bar{\Lambda}}_{22}\right)^{1 / 2} \overline{\bar{\Theta}}_{12} \\
=-\overline{\bar{\Theta}}_{21}^{H}\left(\overline{\bar{I}}-\overline{\bar{\Lambda}}_{11}\right)^{1 / 2} \overline{\bar{U}}_{21}^{H} \overline{\bar{U}}_{22} \overline{\bar{\Lambda}}_{22}^{1 / 2} .
\end{aligned}
$$

Relations (11) also constrain the singular values of the subnetworks. Since $\overline{\bar{S}}_{M}$ is lossless, $\overline{\bar{S}}_{11}$ and $\overline{\bar{S}}_{22}$ must be nonamplifying $\left(0 \leq \lambda_{11, n}, \lambda_{22, n} \leq 1\right)$. Otherwise, an excitation could be found that violates the lossless condition. This observation coupled with the constraints in (11) indicates that $0 \leq \lambda_{12, n}, \lambda_{21, n} \leq 1$ as well. It should also be mentioned that if we also require the matching network to be reciprocal $\left(\overline{\bar{S}}_{M}=\overline{\bar{S}}_{M}^{T}\right.$, where $\{\cdot\}^{T}$ is the transpose operator), additional constraints are placed on the matching network SVD representation.

\section{Optimal ANTENNA Match}

A study of termination-dependent diversity performance would be incomplete without considering an optimal termination that maximizes received power. While such a multiport conjugate match has been discussed in prior work [10], [14]-[16], the SVD analysis proposed here facilitates a significantly simplified proof that $\overline{\bar{S}}_{11}=\overline{\bar{S}}_{S}^{H}$ results in maximum power transfer to the load. We therefore provide here an abbreviated version of this proof.

\section{A. Load Match}

To begin the derivation, we remove the source coupling block in Fig. 1 to arrive at the network in Fig. 2. The power to the load will be maximized for any possible excitation $\left(\bar{a}_{1}\right)$ if and only if we can find $\overline{\bar{S}}_{M}$ for a lossless network such that $\overline{\bar{\Gamma}}_{\text {in }}=\overline{\overline{0}}$.

To show that $\overline{\bar{S}}_{22}=\overline{\bar{S}}_{L}^{H}$ is sufficient to ensure that $\overline{\bar{\Gamma}}_{\text {in }}=\overline{\overline{0}}$ for arbitrary excitation, we use the SVD representations $\bar{S}_{22}=$ $\overline{\bar{S}}_{L}^{H}=\left[\overline{\bar{U}}_{L} \overline{\bar{\Lambda}}_{L}^{1 / 2} \overline{\bar{V}}_{L}^{H}\right]^{H}$ and $\overline{\bar{S}}_{11}=\overline{\bar{U}}_{11} \overline{\bar{\Lambda}}_{11}^{1 / 2} \overline{\bar{V}}_{11}^{H}$. We also make the reasonable assumption that the singular values $\Lambda_{11, n n}<1$ $\left(\overline{\bar{S}}_{11}\right.$ is lossy), since otherwise we could find an input signal that would be completely reflected, violating the zero reflection 


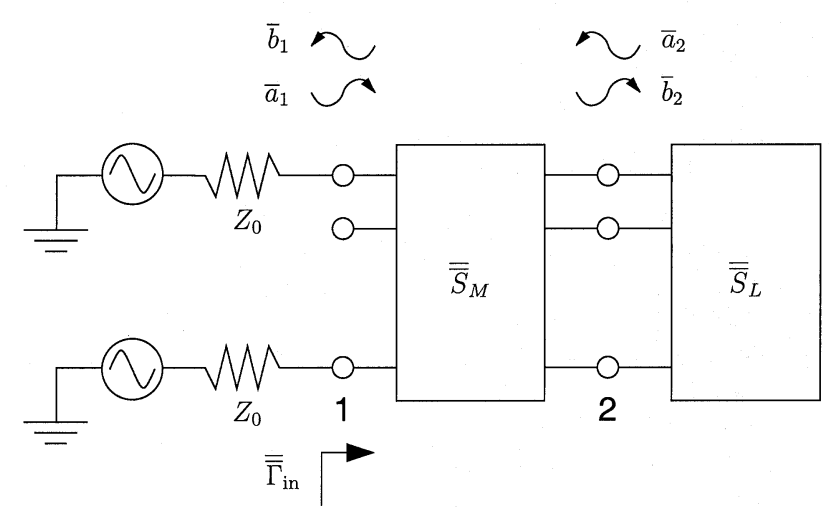

Fig. 2. Setup for the load matching problem. To ensure maximum power transfer, we must find $\overline{\bar{S}}_{M}$ such that $\overline{\bar{\Gamma}}_{\text {in }}=\overline{\overline{0}}$.

condition. Using the constraints in (11) coupled with (5) leads to

$$
\begin{aligned}
\overline{\bar{\Gamma}}_{\text {in }}=\left[\overline{\bar{U}}_{11} \overline{\bar{\Lambda}}_{11}^{1 / 2}\right. & +\overline{\bar{U}}_{12}\left(\overline{\bar{I}}-\overline{\bar{\Lambda}}_{L}\right)^{-1 / 2} \overline{\bar{\Lambda}}_{L}^{1 / 2} \\
& \left.\cdot \overline{\bar{\Theta}}_{12} \overline{\bar{V}}_{L}^{H} \overline{\bar{U}}_{21}\left(\overline{\bar{I}}-\overline{\bar{\Lambda}}_{11}\right)^{1 / 2} \overline{\bar{\Theta}}_{21}\right] \overline{\bar{V}}_{11}^{H} .
\end{aligned}
$$

Since $\overline{\bar{S}}_{L}$ is lossy, we can rewrite (12) for this problem as

$$
\begin{array}{r}
\left(\overline{\bar{I}}-\overline{\bar{\Lambda}}_{L}\right)^{-1 / 2} \overline{\bar{\Lambda}}_{L}^{1 / 2} \overline{\bar{\Theta}}_{12} \overline{\bar{V}}_{L}^{H} \overline{\bar{U}}_{21}\left(\overline{\bar{I}}-\overline{\bar{\Lambda}}_{11}\right)^{1 / 2} \overline{\bar{\Theta}}_{21} \\
=-\overline{\bar{U}}_{12}^{H} \overline{\bar{U}}_{11} \overline{\bar{\Lambda}}_{11}^{1 / 2}
\end{array}
$$

which upon substitution into (13) results in

$$
\overline{\bar{\Gamma}}_{\text {in }}=\left[\overline{\bar{U}}_{11} \overline{\bar{\Lambda}}_{11}-\overline{\bar{U}}_{12} \overline{\bar{U}}_{12}^{H} \overline{\bar{U}}_{11} \overline{\bar{\Lambda}}_{11}\right] \overline{\bar{V}}_{11}^{H}=\overline{\overline{0}} .
$$

Therefore, choosing $\overline{\bar{S}}_{22}=\overline{\bar{S}}_{L}^{H}$ is sufficient to ensure that $\overline{\bar{\Gamma}}_{\text {in }}=$ $\overline{\overline{0}}$ for arbitrary excitation.

Next, we show that $\overline{\bar{\Gamma}}_{\text {in }}=\overline{\overline{0}}$ implies that $\overline{\bar{S}}_{22}=\overline{\bar{S}}_{L}^{H}$, indicating that this latter condition is also necessary for maximizing power transfer. Multiplying (5) by $\overline{\bar{S}}_{12}^{H}$, assuming $\overline{\bar{\Gamma}}_{\text {in }}=\overline{\overline{0}}$, and making appropriate substitutions from the lossless conditions (10), we obtain

$$
\underbrace{\left[-\overline{\bar{S}}_{22}^{H}+\left(\overline{\bar{I}}-\overline{\bar{S}}_{22}^{H} \overline{\bar{S}}_{22}\right) \overline{\bar{S}}_{L}\left(\overline{\bar{I}}_{-} \overline{\bar{S}}_{22} \overline{\bar{S}}_{L}\right)^{-1}\right]}_{\overline{\bar{M}}} \overline{\bar{S}}_{21}=\overline{\overline{0}} .
$$

We now recognize that $\overline{\bar{S}}_{21}$ must be full-rank, since otherwise we would have $\Lambda_{21, n n}=0$ and $\Lambda_{11, n n}=1$ for some $n$ that could lead to complete reflection. Therefore, (16) can only be satisfied if $\overline{\bar{M}}=\overline{\overline{0}}$, which after simplification yields $\overline{\bar{S}}_{22}=$ $\overline{\bar{S}}_{L}^{H}$. The combination of the two preceding proofs indicates that maximum power transfer is achieved if and only if $\overline{\bar{S}}_{22}=\overline{\bar{S}}_{L}^{H}$.

\section{B. Source Match}

We now consider the problem of matching the arbitrary (coupled) source element in Fig. 1 to a set of uncoupled identical loads of impedance $Z_{0}$. In this case, $\overline{\bar{S}}_{L}=\overline{\overline{0}}$ so that $\bar{a}_{2}=\overline{0}$ and
$\overline{\bar{\Gamma}}_{\text {in }}=\overline{\bar{S}}_{11}$. If we collapse the matching network and load network into a single network block, then our equivalent network is in the form of Fig. 2 with $\overline{\bar{S}}_{M}$ replaced by $\overline{\bar{S}}_{c}$ and $\overline{\bar{S}}_{L}$ replaced by the collapsed network with input reflection coefficient $\overline{\bar{S}}_{11}$. Based upon our work above and since the matching network is lossless, we know that all available power will be transfered to the loads if and only if $\overline{\bar{S}}_{11}=\overline{\bar{S}}_{S}^{H}$.

\section{Bilateral Hermitian Match}

To demonstrate the bilateral conjugate match condition, we first set $\overline{\bar{S}}_{L}=\overline{\bar{\Gamma}}_{\text {out }}^{H}$ in (4) and subsequently use (6). Then, assuming that $\overline{\bar{S}}_{12}$ is full-rank (invertible) and applying the lossless conditions in (10) leads to the simplified expression

$$
\overline{\bar{\Gamma}}_{\text {in }}=\overline{\bar{S}}_{S}^{H}\left(\overline{\bar{I}}-\overline{\bar{S}}_{11}^{H} \overline{\bar{S}}_{S}^{H}\right)^{-1}\left(\overline{\bar{I}}-\overline{\bar{S}}_{11}^{H} \overline{\bar{\Gamma}}_{\mathrm{in}}\right) .
$$

Obviously, $\overline{\bar{\Gamma}}_{\text {in }}=\overline{\bar{S}}_{S}^{H}$ is a (unique) solution to this equation. Therefore, $\overline{\bar{\Gamma}}_{\text {out }}=\overline{\bar{S}}_{L}$ implies that $\overline{\bar{\Gamma}}_{\text {in }}=\overline{\bar{S}}_{S}^{H}$.

\section{Unequal Number of Input and Output Ports}

To illustrate the impact of assuming $M \neq N$, consider the load-matching problem with more input ports $(M)$ than output ports $(N)$. Our requirement that $\overline{\bar{S}}_{21}$ be full rank is certainly violated, and therefore we cannot find a matching network that will provide maximum power transfer for arbitrary excitation. However, if input signals are limited to an $N$-dimensional subspace, we can find a linear element that takes signals on the $M$ input ports and converts them to $N$ output ports, and a suitable matching network can then be found. Alternately, if $M<N$ we have no difficulties, since we can simply add input ports that are not connected to the source. Similar arguments exist in the source matching problem.

\section{Termination-DePEndEnt Diversity PERFormance}

\section{A. Received Signal Covariance}

The performance of antenna diversity systems depends upon the signal strength on each antenna branch as well as the signal correlation between branches. To assess these metrics for different degrees of antenna coupling and receiver terminations, we will compute the covariance matrix for the voltages received on each branch. For the network in Fig. 1 with $Z_{0}=1$, the received voltages are given by

$$
\begin{aligned}
\bar{v}_{L} & =\bar{b}_{2}+\bar{a}_{2}=\left(\overline{\bar{I}}+\overline{\bar{S}}_{L}\right) \bar{b}_{2} \\
& =\left(\overline{\bar{I}}+\overline{\bar{S}}_{L}\right)\left(\overline{\bar{I}}-\overline{\bar{S}}_{22} \overline{\bar{S}}_{L}\right)^{-1} \overline{\bar{S}}_{21} \bar{a}_{1}
\end{aligned}
$$

where we have used that $\bar{b}_{2}=\overline{\bar{S}}_{22} \bar{a}_{2}+\overline{\bar{S}}_{21} \bar{a}_{1}$ and $\bar{a}_{2}=\overline{\bar{S}}_{L} \bar{b}_{2}$. Using (3) with $\bar{b}_{1}=\overline{\bar{\Gamma}}_{\text {in }} \bar{a}_{1}$ in (18) leads to

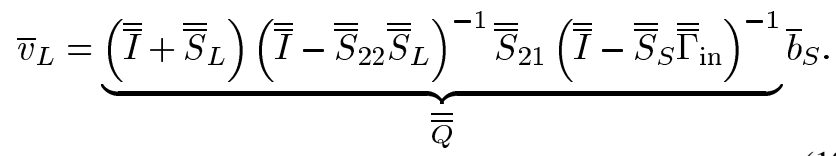

The covariance matrix is therefore given as

$$
\overline{\bar{R}}_{L}=\mathrm{E}\left\{\bar{v}_{L} \bar{v}_{L}^{H}\right\}=\overline{\overline{Q R}}_{S} \overline{\bar{Q}}^{H}
$$


where $\overline{\bar{R}}_{S}=E\left\{\bar{b}_{S} \bar{b}_{S}^{H}\right\}$ is the covariance of $\bar{b}_{S}$ and $E\{\cdot\}$ represents an expectation. The form of $\overline{\bar{Q}}$ in (19) represents the general voltage transfer function and is relatively complicated. However, for cases of practical interest, this expression is significantly simplified, as demonstrated in Section IV-C and -D.

To relate $\bar{b}_{S}$ to the physical antenna properties, consider an antenna array consisting of $N$ arbitrary antenna elements embedded in a reciprocal medium. The far-field radiation pattern of the array is

$$
\bar{E}(\Omega)=\sum_{n} \bar{F}_{n}(\Omega) i_{n}
$$

where $i_{n}$ is the current on the $n$th element, $\bar{F}_{n}(\Omega)$ is the vector far-field radiation pattern of the $n$th element when all other elements are open-circuited $\left(i_{k}=0\right.$ for $\left.k \neq n\right)$, and $\Omega$ represents a direction in solid angle. We may stack all of the far-field patterns (one per column) into a single matrix to obtain

$$
\bar{E}(\Omega)=\overline{\bar{F}}(\Omega) \bar{i}
$$

Now, assume a plane wave arrives from the solid angle direction $\Omega_{0}$ (propagating in the $-\Omega_{0}$ direction) with complex field strength $E_{0}=A \exp (j \phi)(A$ real $)$ and electric field polarization vector $\hat{e}$. If the antennas are open-circuited, then by reciprocity the voltages on the antenna elements are given by

$$
\bar{v}=2 c_{1} E_{0} \overline{\bar{F}}\left(\Omega_{0}\right)^{T} \hat{e}
$$

where $c_{1}$ is a complex constant. Equating this open-circuit antenna response to the voltage obtained assuming that port 1 in Fig. 1 is open-circuited, we arrive at the expression

$$
\bar{b}_{S}=c_{1} E_{0}\left(\overline{\bar{I}}-\overline{\bar{S}}_{S}\right) \overline{\bar{F}}\left(\Omega_{0}\right)^{T} \hat{e}=c_{1} E_{0} \overline{\bar{F}}^{\prime}\left(\Omega_{0}\right)^{T} \hat{e}
$$

where we have included the impedance mismatch factor $\left(\overline{\bar{I}}-\overline{\bar{S}}_{S}\right)$ in the effective radiation pattern $\overline{\bar{F}}^{\prime}\left(\Omega_{0}\right)$ for later simplicity.

We emphasize here that the choice of using element patterns computed with all other elements open-circuited is only one of a variety of possibilities. For example, we could have chosen to compute patterns with all other ports terminated in the system impedance $Z_{0}$. In this case, however, the superposition in (22) and formulation leading to (24) would need to change appropriately.

The covariance of $\bar{b}_{S}$ may now be written explicitly as

$$
\overline{\bar{R}}_{S}=\left|c_{1}\right|^{2} \int d \Omega d \hat{e} d A p(\Omega, \hat{e}, A) A^{2} \overline{\bar{F}}^{\prime}(\Omega)^{T} \hat{e} \hat{e}^{T} \overline{\bar{F}}^{*}(\Omega)
$$

where $p(\Omega, \hat{e}, A)$ represents the probability density of the incident field angle of arrival, polarization, and amplitude. For the following, we will make the standard assumptions that:

1) the antennas and incident waves have the same single polarization;

2) arrival angles are restricted to and uniformly distributed on a solid angle sector $\Delta \Omega$;

3) field amplitudes are independent of arrival angle and have a variance $E^{2}$.
Under these contraints, we have

$$
\begin{aligned}
\overline{\bar{R}}_{S}^{*} & =\left|c_{1}\right|^{2} E^{2} \frac{\int_{\Delta \Omega} d \Omega \overline{\bar{F}}^{\prime H}(\Omega) \overline{\bar{F}}^{\prime}(\Omega)}{\int_{\Delta \Omega} d \Omega} \\
& =c_{2} \overline{\bar{P}}
\end{aligned}
$$

where $c_{2}=\left(\left|c_{1}\right|^{2} E^{2}\right) / \int_{\Delta \Omega} d \Omega$. The covariance of $\bar{b}_{L}$ can now be written as

$$
\overline{\bar{R}}_{L}=c_{2} \overline{\bar{Q}} \overline{\bar{P}}^{*} \overline{\bar{Q}}^{H}
$$

In some cases, a more descriptive statistical model of the channel is available (such as a path-based model [17]-[19]). In this case, $\bar{b}_{S}$ can be computed from (24), with $E_{0}$ and $\hat{e}$ obtained from the model, for a set of Monte Carlo channel realizations. The covariance $\overline{\bar{R}}_{S}=\mathrm{E}\left\{\bar{b}_{S} \bar{b}_{S}^{H}\right\}$ can then be constructed using a sample mean to estimate the expectation.

\section{B. Source Covariance for Full Angular Spread}

Under the special case that the multipath arrival sector $\Delta \Omega$ extends over the full angular range of the propagation environment, a simplification can be introduced. To see this, consider the array operating as a transmitter. Using (22), the power radiated by the array is given as

$$
\begin{aligned}
P_{\text {rad }} & =c_{3} \oint d \Omega\|\bar{E}(\Omega)\|^{2} \\
& =c_{3} \bar{i}^{H}\left[\oint d \Omega \overline{\bar{F}}^{H}(\Omega) \overline{\bar{F}}(\Omega)\right] \bar{i}
\end{aligned}
$$

where $c_{3}$ is a real constant. From (1) with $Z_{0}=1$, we know that $\bar{i}=\left(\overline{\bar{I}}-\overline{\bar{S}}_{S}\right) \bar{a}$, where in this case $\bar{a}$ represents the voltage waves incident on the antenna ports. The radiated power becomes

$$
\begin{aligned}
P_{\text {rad }} & =c_{3} \bar{a}^{H}\left(\overline{\bar{I}}-\overline{\bar{S}}_{S}\right)^{H}\left[\oint d \Omega \overline{\bar{F}}^{H}(\Omega) \overline{\bar{F}}(\Omega)\right]\left(\overline{\bar{I}}-\overline{\bar{S}}_{S}\right) \bar{a} \\
& =c_{3} \bar{a}^{H} \underbrace{\left[\oint d \Omega \overline{\bar{F}}^{H}(\Omega) \overline{\bar{F}}^{\prime}(\Omega)\right]}_{\overline{\bar{P}}} \bar{a} .
\end{aligned}
$$

For a lossless antenna, the power radiated is equal to the power delivered to the antenna network as expressed in (8). Equating (29) and (8) and using the result in (26) therefore leads to the source covariance

$$
\overline{\bar{R}}_{S}=c_{2} \overline{\bar{P}}^{*}=c\left(\overline{\bar{I}}-\overline{\bar{S}}_{S}^{H} \overline{\bar{S}}_{S}\right)^{*}
$$

where $c=c_{2} / c_{3}$. If the antenna and transmission media are reciprocal, then $\overline{\bar{S}}_{S}=\overline{\bar{S}}_{S}^{T}$, resulting in

$$
\overline{\bar{R}}_{S}=c\left(\overline{\bar{I}}-\overline{\bar{S}}_{S} \overline{\bar{S}}_{S}^{H}\right) \text {. }
$$

The key observation concerning (31) is that under the propagation conditions outlined, the covariance matrix can be computed without resorting to integration of the radiation patterns, as previously observed in [10].

\section{Simplifications for Practical Terminations}

The general framework for computing the covariance matrix of terminated coupled antennas is simplified for several practical terminations. In this section, we consider the cases of 
terminating the antenna ports with open-circuit characteristic impedance and self-impedance matched loads. For all cases except for open-circuited terminations, we use the (realistic) load network consisting of one resistor of value $Z_{0}=1$ on each port, leading to $\overline{\bar{S}}_{L}=\overline{\overline{0}}$. Since this results in $\overline{\bar{\Gamma}}_{\text {in }}=\overline{\bar{S}}_{11}$, the matching network is used to create the desired antenna port terminations.

1) Open-Circuit Terminations: For open-circuit termination, the matching network is removed $\overline{\bar{S}}_{11}=\overline{\bar{S}}_{22}=0$, $\left.\overline{\bar{S}}_{12}=\overline{\bar{S}}_{21}=\overline{\bar{I}}\right)$ such that $\overline{\bar{\Gamma}}_{\text {in }}=\overline{\bar{S}}_{L}=\overline{\bar{I}}$. Under these conditions, the covariance matrix simplifies to

$$
\overline{\bar{R}}_{L, \mathrm{oc}}=4\left(\overline{\bar{I}}-\overline{\bar{S}}_{S}\right)^{-1} \overline{\bar{R}}_{S}\left(\overline{\bar{I}}-\overline{\bar{S}}_{S}^{H}\right)^{-1} .
$$

2) Characteristic Impedance Terminations: In this case, we again remove the matching network but use $\overline{\bar{\Gamma}}_{\text {in }}=\overline{\overline{0}}$. This results in $\overline{\bar{Q}}=\overline{\bar{I}}$ such that

$$
\overline{\bar{R}}_{L, Z_{0}}=\overline{\bar{R}}_{S} .
$$

3) Self-Impedance Match: Self-impedance match refers to the condition where port $n$ is terminated in the self-impedance of antenna $n$. In this case, $\overline{\bar{\Gamma}}_{\text {in }}=\overline{\bar{S}}_{11}=\operatorname{diag}\left\{\overline{\bar{S}}_{S}^{*}\right\}$, where the $\operatorname{diag}\{\cdot\}$ operator creates a diagonal matrix from the diagonal entries of the operand. The resulting covariance matrix becomes

$$
\overline{\bar{R}}_{L, \text { self }}=\overline{\bar{S}}_{21}\left(\overline{\bar{I}}-\overline{\bar{S}}_{S} \overline{\bar{S}}_{11}\right)^{-1} \overline{\bar{R}}_{S}\left(\overline{\bar{I}}-\overline{\bar{S}}_{S} \overline{\bar{S}}_{11}\right)^{H(-1)} \overline{\bar{S}}_{21}^{H} \text {. }
$$

\section{Optimal Hermitian Match}

As a framework for analysis, let $\overline{\bar{\Gamma}}_{\text {in }}=\overline{\bar{S}}_{11}=\overline{\bar{S}}_{S}^{H}$, which leads to the covariance matrix

$$
\overline{\bar{R}}_{L, \mathrm{opt}}=\overline{\bar{S}}_{21}\left(\overline{\bar{I}}-\overline{\bar{S}}_{S} \overline{\bar{S}}_{S}^{H}\right)^{-1} \overline{\bar{R}}_{S}\left(\overline{\bar{I}}-\overline{\bar{S}}_{S} \overline{\bar{S}}_{S}^{H}\right)^{-1} \overline{\bar{S}}_{21}^{H} .
$$

Using the conditions for lossless matching networks from Section II-C, the two relevant blocks of the matching network are represented using

$$
\begin{aligned}
& \overline{\bar{S}}_{11}=\overline{\bar{S}}_{S}^{H}=\overline{\overline{V \Lambda}}^{1 / 2} \overline{\bar{U}}^{H} \\
& \overline{\bar{S}}_{21}=\overline{\bar{U}}_{21}(\overline{\bar{I}}-\overline{\bar{\Lambda}})^{1 / 2} \overline{\bar{\Theta}}_{21} \overline{\bar{U}}^{H} .
\end{aligned}
$$

These conditions transform (35) to the form

$$
\begin{aligned}
\overline{\bar{R}}_{L, \mathrm{opt}}=\overline{\bar{U}}_{21} \overline{\bar{\Theta}}_{21}(\overline{\bar{I}}-\overline{\bar{\Lambda}})^{-1 / 2} \overline{\bar{U}}^{H} \overline{\bar{R}}_{S} \overline{\bar{U}} \\
\times(\overline{\bar{I}}-\overline{\bar{\Lambda}})^{-1 / 2} \overline{\bar{\Theta}}_{21}^{H} \overline{\bar{U}}_{21}^{H} .
\end{aligned}
$$

Under the conditions considered in Section IV-B where multipath components arrive uniformly from all angles of arrival, we use (31) along with (36) to obtain

$$
\overline{\bar{R}}_{S}=c \overline{\bar{U}}(\overline{\bar{I}}-\overline{\bar{\Lambda}}) \overline{\bar{U}}^{H} \text {. }
$$

Placing this result in (37) yields the simplified expression

$$
\overline{\bar{R}}_{L, \mathrm{opt}}=c \overline{\bar{U}}_{21} \overline{\bar{\Theta}}_{21} \overline{\bar{\Theta}}_{21}^{H} \overline{\bar{U}}_{21}^{H}=c \overline{\bar{I}} .
$$

This result implies that under these circumstances, the optimal Hermitian match perfectly decorrelates the signals on the loads.
Other studies have noted the reduced correlation associated with termination [3], [6]-[12], but have not provided a framework for arriving at this perfectly diagonal covariance. It is important to realize that this decorrelation is simply the result of recombination of the received signals, and therefore does not necessarily enhance the diversity performance of the system. Nevertheless, it is interesting to be able to mathematically predict this behavior using the proposed analysis approach.

When full angular spread does not exist, the optimal matching network can be further specified to diagonalize the covariance matrix. Consider again (37) and let

$$
\begin{aligned}
\overline{\bar{T}} & =(\overline{\bar{I}}-\overline{\bar{\Lambda}})^{-1 / 2} \overline{\bar{U}}^{H} \overline{\bar{R}}_{S} \overline{\bar{U}}(\overline{\bar{I}}-\overline{\bar{\Lambda}})^{-1 / 2} \\
& =\overline{\bar{U}}_{T} \overline{\bar{\Lambda}}_{T} \overline{\bar{U}}_{T}^{H}
\end{aligned}
$$

where (41) represents the eigenvector decomposition of $\overline{\bar{T}}$. Since the product $\overline{\bar{U}}_{21} \overline{\bar{\Theta}}_{21}$ is arbitrary as long as it is unitary, we can choose $\overline{\bar{U}}_{21} \overline{\bar{\Theta}}_{21}=\overline{\bar{U}}_{T}^{H}$ to obtain

$$
\overline{\bar{R}}_{L, \mathrm{opt}}=\overline{\bar{\Lambda}}_{T}
$$

the diagonal matrix of eigenvalues of $\overline{\bar{T}}$. In the case of two vertically oriented dipoles, the problem symmetry leads to $2 \times 2$ real Toeplitz symmetric matrices that share common eigenvectors. The result is that the diagonalization can be accomplished by choosing $\overline{\bar{U}}_{21}=\overline{\bar{I}}$.

\section{COMPUTATIONAL EXAMPLES}

To demonstrate application of the analysis framework developed in this paper and to illustrate the impact of termination on the diversity performance of mutually coupled antennas, we will explore a receive array consisting of two coupled dipoles. While closed-form expressions for coupled dipole impedance matrices exist (for reasonable antenna spacings), expressions for the patterns do not, motivating the use of full-wave electromagnetic solutions. Furthermore, simple thin-wire simulations assume that the current does not vary in azimuth around the wire, an assumption that is violated for very closely spaced dipoles [20]. The antenna coupled impedance computations are particularly sensitive to this current variation, and the computation essentially breaks down as the antenna spacing is reduced to zero [21], [22].

In this paper, it is desired to characterize the coupled antennas as the spacing is reduced to zero. As a result, we have chosen to use the finite-difference time-domain (FDTD) method [5], [23] to perform detailed simulations that return both S-parameter and radiation pattern descriptions for the dipole antennas. In this analysis, the $z$-oriented half-wave (total-length) dipoles with wire radius $0.01 \lambda$ and separated by a distance $d$ are located at the center of the computational domain. Because we are considering narrow-band systems, single-frequency antenna excitation is used. The FDTD grid uses 80 cells per wavelength in the $z$ direction and 200 cells per wavelength in the $x$ and $y$ directions. This finer resolution is required to adequately model the current variations in the azimuthal direction on the finite-radius wire for close antenna spacings.

Because of the fine grid resolution, a relatively small buffer region of only a quarter-wavelength (to minimize simulation memory) is placed between the antennas and the terminating 


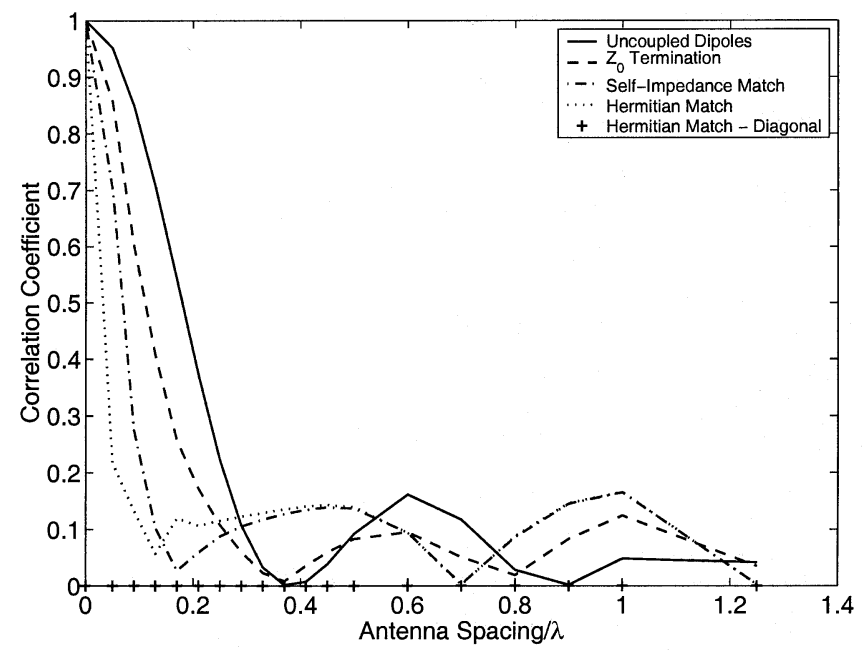

Fig. 3. Plot of branch signal correlation as a function of antenna spacing for two coupled dipole antennas terminated with various loads. The result for independent dipoles is shown for comparison.

eight-cell perfectly matched layer absorbing boundary condition. The impact of this small buffer region was investigated by reducing the $x$ and $y$ resolution to 100 cells per wavelength and comparing impedances and radiation patterns for half- and quarter-wavelength buffer thicknesses with a dipole spacing of $0.13 \lambda$. The resulting fractional change in self- and mutual impedances was only $1.4 \times 10^{-4}$ and $1.9 \times 10^{-4}$, respectively. The maximum fractional change in the radiated electric field intensity when a single antenna was excited was $1.5 \times 10^{-4}$.

Based upon the formulation in Section IV-A, pattern computations are performed when one antenna is excited while the second is terminated in an open circuit. The antenna S-parameter matrix $\overline{\bar{S}}_{S}$ is computed with the antennas terminated in $Z_{0}$. Multipath arrivals are assumed confined to the horizontal plane in this paper. Unless otherwise specified, arrival angles are uniformly distributed within this plane $(0 \leq \phi<2 \pi)$. As a result, the radiation patterns are normalized such that

$$
\int_{0}^{2 \pi} P\left(\theta=\frac{\pi}{2} \phi\right) d \phi=1
$$

where $P(\theta, \phi)$ is the antenna power pattern. This same normalization is applied to an array of ideal (uncoupled) dipoles used as a baseline for defining the degree of diversity performance.

As a first example, it is interesting to compute the correlation coefficient of the signals on the two antennas. This is simply derived from the covariance matrix using

$$
\rho=\frac{R_{L, 12}}{\sqrt{R_{L, 11} R_{L, 22}}}
$$

where $R_{L, i j}$ represents the $i, j$ th element of the computed load covariance matrix. Fig. 3 plots the variation of this quantity as a function of antenna spacing for the different terminations considered in this work. For the optimal match, the matrix $\overline{\bar{U}}_{21}$ is chosen as $\overline{\bar{U}}_{11}$ and $\overline{\bar{I}}$ for the cases where $\overline{\bar{R}}_{L}$ is not diagonal and diagonal, respectively. The reduced correlation afforded by proper termination is clearly apparent in these results. It is noteworthy that all terminations result in reduced correlation as compared with the result obtained for uncoupled

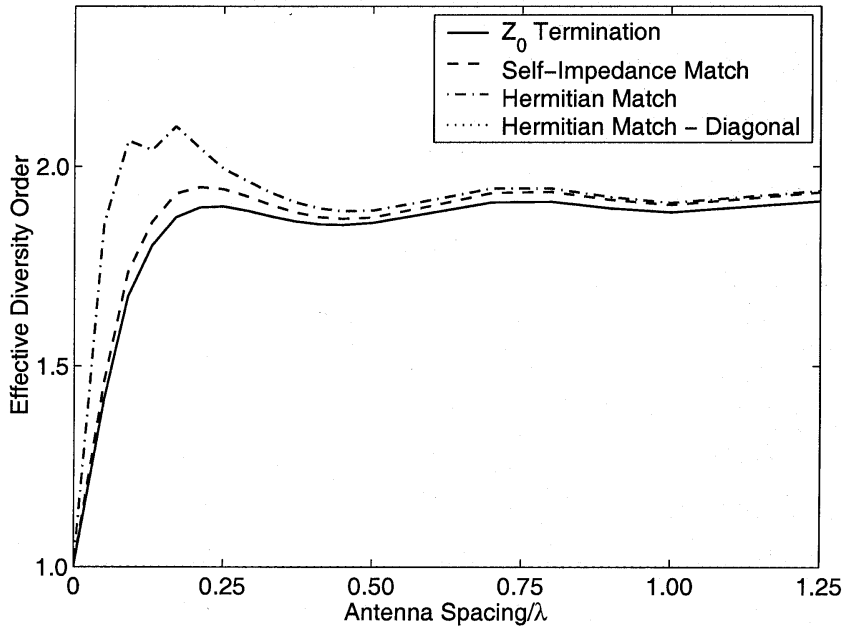

Fig. 4. Plot of effective diversity order as a function of antenna spacing for two coupled dipole antennas terminated with various loads. Correlations are computed assuming arrivals are equally likely to appear at any angle within the horizontal plane. The two Hermitian match results lie right on top of each other.

dipoles, confirming the results of other research [7], [8], [12]. However, it is important to point out that this study has included the effects of the mutual impedance and terminations in the analysis and provides a comprehensive examination of this effect for a variety of termination types.

Naturally, the correlation coefficient provides only a partial indication of the diversity performance of multiple antennas, since the power on each branch is also an important factor. In order to investigate the full diversity benefit, we utilize the concept of effective diversity order introduced in [9]. In this metric, the diversity benefit is measured in comparison to what is possible using two equal power and uncoupled antennas. For this paper, the diversity order is determined from the data at the $1 \%$ level on the diversity cumulative distribution functions and assuming maximal ratio combining. For the coupled antennas, diversity performance is computed using the eigenvalues of the covariance matrix to represent two independent branches with unequal average signal-to-noise ratio (SNR). Full details on this metric are provided in [9].

Fig. 4 shows the diversity order as a function of spacing for our different termination conditions. As can be seen, for small antenna spacings, improved matching leads to improved diversity performance. Most striking is the fact that the optimal matching circuits lead to better performance than what is obtainable with independent, equal power branches for small antenna spacings (characterized as a diversity order $>2$ ). This surprising result stems from the fact that optimally matched coupled antennas can actually capture more power than can be collected by two independent dipoles. One reasonable explanation for this increased effective aperture is that a portion of the power scattered by each receiving antenna can be recaptured by the adjacent antenna. This metric also reveals the expected result that although the matching network can diagonalize the covariance matrix, this diagonalization comes at the expense of unequal branch SNR and therefore does not facilitate additional diversity gain. For this reason, the two Hermitian match results lie on top of each other in the plot. For 


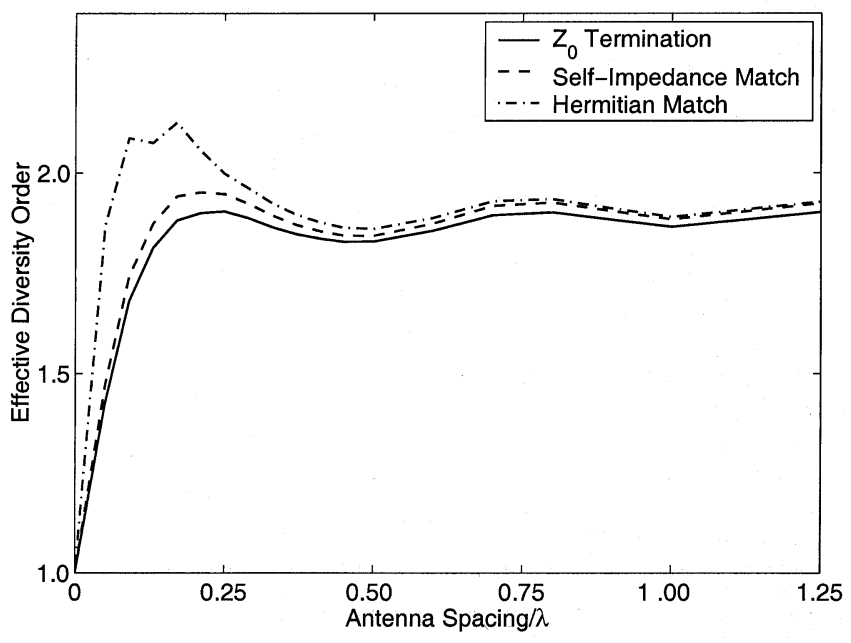

Fig. 5. Plot of effective diversity order as a function of antenna spacing for two coupled dipole antennas terminated with various loads. Correlations are computed from Monte Carlo simulations using a statistical path-based propagation model.

larger spacings, clearly the match becomes less important, as the curves for the different terminations tend to the same value.

Finally, Fig. 5 shows the diversity order as a function of spacing for when the channel is obtained using a statistical path-based channel model [19]. This model, which provides the angles and times of arrival for each individual multipath, structures these arrivals as clusters in space and time [17], [18]. In the simulations, 5000 channel realizations are used to estimate the covariance matrix $\overline{\bar{R}}_{S}$ for each antenna separation. While there certainly are some slight differences between the results in Figs. 4 and 5, the main conclusions obtained from this more practical example are the same as those drawn from the more simplistic, previous computation. Note that one explanation for this similarity is that when 5000 channels are considered, the statistical distribution of the arrival angles tends to be uniform despite the clustered nature of a single realization.

\section{CONCLUSION}

This paper has presented a new analysis of multiport matching networks, applicable to the mitigation of mutual coupling in compact antenna arrays. The SVD of the S-parameter matrices describing the coupled antenna/termination network leads to a powerful tool that facilitates specification and analysis of the network response. Additionally, this framework allows formulation of a new, simplified proof of the optimal (Hermitian) match condition for coupled networks and leads to relatively straightforward expressions for the covariance matrix of signals received at the antenna terminations. These results were used in conjunction with electromagnetic analysis of coupled dipole antennas to demonstrate the potential diversity benefit offered by two-element arrays for different possible termination conditions. The results revealed that for close antenna spacing as might be encountered on portable devices, proper matching plays a noticeable role in determining the system performance. Perhaps more importantly, this framework provides a comprehensive analysis tool for characterizing and analyzing the performance of arbitrary coupled antenna systems, including all three relevant aspects of radiation pattern, mutual impedance, and antenna termination.

\section{REFERENCES}

[1] W. C. Jakes, Microwave Mobile Communications. New York: IEEE Press, 1993.

[2] C. A. Balanis, Antenna Theory: Analysis and Design: Wiley, 1997.

[3] K. Boyle, "Radiation patterns and correlation of closely spaced linear antennas," IEEE Trans. Antennas Propagat., vol. 50, pp. 1162-1165, Aug. 2002.

[4] S. C. K. Ko and R. D. Murch, "Compact integrated diversity antenna for wireless communications," IEEE Trans. Antennas Propagat., vol. 49, pp. 954-960, June 2001.

[5] M. A. Jensen and Y. Rahmat-Samii, "Performance analysis of antennas for hand-held transceivers using FDTD," IEEE Trans. Antennas Propagat., vol. 42, pp. 1106-1113, Aug. 1994.

[6] M. C. Leifer, "Signal correlations in coupled cell and MIMO antennas," in Proc. IEEE Antennas and Propagation Society Int. Symp., vol. 3, San Antonio, TX, June 16-21, 2002, pp. 194-197.

[7] J. Luo, J. R. Zeidler, and S. McLaughlin, "Performance analysis of compact antenna arrays with MRC in correlated nakagami fading channels," IEEE Trans. Veh. Technol., vol. 50, pp. 267-277, Jan. 2001.

[8] T. Svantesson and A. Ranheim, "Mutual coupling effects on the capacity of multielement antenna systems," in Proc. IEEE ICASSP'2001, vol. 4, Salt Lake City, UT, May 7-11, 2001, pp. 2485-2488.

[9] O. Nørklit, P. D. Teal, and R. G. Vaughan, "Measurement and evaluation of multi-antenna handsets in indoor mobile communication," IEEE Trans. Antennas Propagat., vol. 49, pp. 429-437, Mar. 2001.

[10] R. G. Vaughan and J. B. Andersen, "Antenna diversity in mobile communications,” IEEE Trans. Veh. Technol., vol. VT-36, pp. 147-172, Nov. 1987.

[11] C. Waldschmidt, J. v. Hagen, and W. Wiesbeck, "Influence and modeling of mutual coupling in MIMO and diversity systems," in Proc. IEEE Antennas and Propagation Society Int. Symp., vol. 3, San Antonio, TX, June 16-21, 2002, pp. 190-193.

[12] R. G. Vaughan and N. L. Scott, "Closely spaced monopoles for mobile communications," Radio Sci., vol. 28, pp. 1259-1266, Nov.-Dec. 1993.

[13] D. M. Pozar, Microwave Engineering. New York: Wiley, 1998.

[14] J. L. Allen and B. L. Diamond, "Mutual coupling in array antennas," Lincoln Laboratory, MIT, Tech. Rep. 424 (ESD-TR-66-443), 1966.

[15] R. A. Speciale, "Advanced design of phased-array beam-forming networds," IEEE Antennas Propagat. Mag., vol. 38, pp. 22-34, Aug. 1996.

[16] H. A. Haus and R. B. Adler, Circuit Theory of Linear Noisy Networks. New York: Wiley, 1959

[17] A. A. M. Saleh and R. A. Valenzuela, "A statistical model for indoor multipath propagation," IEEE J. Select. Areas Commun., vol. SAC-5, pp. 128-137, Feb. 1987.

[18] Q. H. Spencer, B. D. Jeffs, M. A. Jensen, and A. L. Swindlehurst, "Modeling the statistical time and angle of arrival characteristics of an indoor multipath channel," IEEE J. Select. Areas Commun., vol. 18, pp. 347-360, Mar. 2000.

[19] J. W. Wallace and M. A. Jensen, "Modeling the indoor MIMO wireless channel," IEEE Trans. Antennas Propagat., vol. 50, pp. 591-599, May 2002.

[20] A. C. Ludwig, "Wire-grid modeling of surfaces," IEEE Trans. Antennas Propagat., vol. AP-35, pp. 1045-1048, Sept. 1987.

[21] C. W. Trueman and S. J. Kubina, "Verifying wire-grid model integrity with program "CHECK," Appl. Comput. Electromagn. Soc. J., vol. 5, no. 2, p. 17, Winter 1990.

[22] J. W. Rockway, J. C. Logan, D. W. S. Tam, and S. T. Li, The MININEC System: Microcomputer Analysis of Wire Antennas. Norwood, MA: Artech House, 1988.

[23] A. Taflove and S. C. Hagness, Computational Electrodynamics: The Finite-Difference Time-Domain Method, 2nd ed. Norwood, MA: Artech House, 2000. 
Jon W. Wallace (S'99-M'03) received the B.S. (summa cum laude) and Ph.D. degrees in electrical engineering from Brigham Young University (BYU), Provo, UT, in 1997 and 2002, respectively.

From 1995 to 1997, he was an Associate of Novell, Incorporated, Provo. During 1997, he was a Member of Technical Staff for Lucent Technologies, Denver, CO. From 1998 to 2002, he was a Graduate Student Researcher in the BYU Wireless Communications Laboratory. He is currently a Research Associate of BYU Wireless Communications Laboratory, Vienna, Austria, pursuing joint research with the Technical University of Vienna Mobile Communications Group. His research interests include wireless channel sounding and modeling, optical device modeling, and remote sensing.

Dr. Wallace received the National Science Foundation Graduate Fellowship in 1998
Michael A. Jensen (S'93-M'95-SM'01) received the B.S. (summa cum laude) and M.S. degrees from Brigham Young University (BYU), Provo, UT, in 1990 and 1991, respectively, and the Ph.D. degree from the University of California, Los Angeles (UCLA), in 1994, all in electrical engineering.

From 1989 to 1991, he was a Graduate Research Assistant in the Lasers and Optics Laboratory, BYU. From 1991 to 1994, he was a Graduate Student Researcher in the Antenna Laboratory, UCLA. Since 1994, he has been with the Electrical and Computer Engineering Department, BYU, where he is currently an Associate Professor. His main research interests include antennas and propagation for personal communications, microwave circuit design, radar remote sensing, numerical electromagnetics, and optical fiber communications.

Dr. Jensen is a Member of Eta Kappa Nu and Tau Beta Pi. In 1990, he received a National Science Foundation Graduate Fellowship. He currently is on the Joint Meetings Committee for the IEEE Antennas and Propagation Society. He was the Technical Program Chair for the 2000 IEEE International Symposium on Antennas and Propagation. He received the Best Paper Award from the 1994 IEEE International Symposium on Antennas and Propagation. 